\title{
The Effect of Mobility-induced Location Errors on Geographic Routing in Ad Hoc Networks: Analysis and Improvement using Mobility Prediction
}

\author{
Dongin Son, Ahmed Helmy, Bhaskar Krishnamachari \\ Electrical Engineering, University of Southern California, \{dongjins, helmy, bkrishna\}@usc.edu
}

\begin{abstract}
Geographic routing in mobile ad hoc networks has proved to provide drastic performance improvement over strictly address-centric routing schemes. While geographic routing has been shown to be correct and efficient when location information is accurate, its performance in the face of location errors is not well understood. In this paper, we study the effect of inaccurate location information caused by node mobility under a rich set of scenarios and mobility models. We identify two main problems, named LLNK and LOOP, that are caused by mobility-induced location errors. Based on analysis via $n s-2$ simulations, we propose two mobility prediction schemes --- neighbor location prediction (NLP) and destination location prediction (DLP) to mitigate these problems. Simulation results show noticeable improvement under all mobility models used in our study. Our schemes achieve up to $27 \%$ improvement in packet delivery and $37 \%$ reduction in network resource wastage on average without incurring any additional communication or intense computation.
\end{abstract}

\section{Introduction}

In anticipation of broader use of global positioning system (GPS) [1] and other localization schemes, geographic routing is becoming a very attractive choice for routing in mobile ad hoc networks and also in sensor networks. Geographic routing protocols $[2,3,4,5]$ have been proposed and proved to provide drastic performance improvement over existing ad hoc routing protocols $[6,7,8,9]$. Not only the benefits attained from using geographic routing protocol but the location information itself is important and necessary for many applications.

In geographic routing, the packet forwarding decision is solely based on the location information of neighbors and a destination node at the moment of forwarding. Geographic routing protocols have been shown to be correct and efficient with exact location information. The effect of location errors on geographic routing, however, has not been studied before to our knowledge. This effect is exacerbated with node mobility and harder to resolve because each node may have a different level of location error according to its mobility level. Most previous studies on geographic routing have used the random waypoint mobility model that ignores movement correlation among nodes.

In this study we provide the first study to (1) understand the effect of inaccurate location information caused by node mobility on geographic routing protocols under various mobility models, and (2) provide remedies for the identified problems with a suggested mobility prediction scheme.

We examine the following three main factors that greatly affect the performance of geographic routing protocols:

(a) Freshness of location information: It is not possible to avoid the time gap between the measurement of a location and the time when the information is actually looked up for routing decision, for both proactive and reactive routing protocols, due to the time spent for the delivery of location information and the time passed before the received information is used.

(b) Speed of the mobile nodes: Each mobile node can move at a different speed and the maximum node speed is another critical factor deciding the level of inaccuracy.

(c) The mobility pattern of mobile nodes: If the node movement exhibits a different pattern, the effect of node mobility on the geographic routing protocol will be different. Four different mobility models [10] are adopted in our work: Random waypoint $(R W P)$, Freeway $(F W Y)$, Manhattan $(M H)$ and Reference Point Group Mobility (RPGM).

Based on the simulation results, two major problem types are identified and discussed in this paper: Lost Link (LLNK) problem and LOOP problem. The LLNK problem is related to the link connection problem with neighboring nodes, and the LOOP problem is related to the inaccurate location information of destination nodes caused by their mobility.

We present two mobility prediction (MP) schemes to address these problems: neighbor location prediction (NLP) and destination location prediction (DLP). We find that the performance of geographic routing is significantly increased with MP without any added communication overhead.

We evaluate our proposed schemes through $n s-2$ simulations of the greedy perimeter stateless routing protocol, GPSR $[2,11]$, using the IMPORTANT [10] mobility tool.

The rest of the paper is organized as follows. In section 2, we provide background GPSR and mobility models used. In section 3, the effect of node mobility on geographic routing is discussed based on simulation results. In section 4, two mobility-induced problems are identified. In section 5, mobility prediction scheme is introduced. In section 6, performance improvement is presented. Section 7 concludes the paper.

\section{Background}

In this section we provide a brief description of the geographic routing and mobility models used in our study.

\subsection{Greedy Perimeter Stateless Routing (GPSR)}

GPSR $[2,11]$ is a location-based routing protocol for wireless networks, and it consists of two packet forwarding modes: greedy packet forwarding and perimeter forwarding. The originator of the data generates a packet that contains the coordinates of the destination node. Initially, the packet is forwarded by greedy packet forwarding in which each node makes a localized routing decision based on the location information of its neighbor nodes as follows. Every node periodically broadcast a beacon packet within its own radio range which carries a node-id and current location information. 
Every node which receives a beacon packet stores received information in the neighbor list. Every time a node forwards a packet, it calculates the distances from every neighbor node to the destination node. The neighbor node located closest to the destination node is selected as a next hop. With this localized routing decision, a packet can be delivered to the destination through the optimal path in the distance aspect. However, there are some situations called local maximum where a node cannot find any node located closer to the destination while there exist a detour through a neighbor located further from the destination than itself.

When a node finds out a local maximum situation, the packet forwarding mode is changed to perimeter forwarding. Packets are traversed along the planar graph [2] until it reaches a node that is closer to the destination than the node where greedy forwarding was failed.

\subsection{Mobility Models}

We adopt a rich set of mobility models for our study. Some of the mobility patterns apart from the Random Waypoint (RWP) model that have been studied include the Freeway, RPGM and Manhattan. Each of these was chosen to replicate certain mobile node characteristics not previously captured by the RWP model.

2.2.1. RPGM. Each group of nodes has a group leader that determines the group's motion behavior. Initially, each member of the group is uniformly distributed in the neighborhood of the group leader. Every node has a speed and direction that is derived by randomly deviating slightly from that of the group leader. The speed deviation is set according to the speed deviation ratio $(S D R)$, and the angle deviation ratio is set according to the angle deviation ratio $(A D R)$ as follows.

$$
\begin{gathered}
\left|\vec{V}_{\text {node }}(t)\right|=\left|\vec{V}_{\text {reference }}(t)\right|+\text { random }() \times S D R \times V_{\max } \\
\theta_{\text {node }}(t)=\theta_{\text {reference }}(t)+\text { random }() \times A D R \times \theta_{\max }
\end{gathered}
$$

In our study, we take $S D R=A D R=0.1$.

2.2.2. Freeway Mobility Model. This model emulates the motion behavior of mobile nodes on a freeway (example of shown in figure 1). Each mobile node is restricted to its lane on the freeway and the velocity is temporally dependent on its previous velocity. For two mobile nodes on the same freeway lane within the safety distance, the velocity of the following node cannot exceed the velocity of preceding node. Due to the above relationships, the Freeway mobility pattern is expected to have spatial dependence and high temporal dependence.

2.2.3. Manhattan Mobility Model. The Manhattan model emulates movement of mobile nodes on streets (example shown in figure 2). The mobile node is allowed to move along the grid of horizontal and vertical streets on the map. At an intersection the mobile node can turn left, right or go straight, with probability $0.25,0.25$ and 0.5 , respectively. Velocity of a node at a time slot is dependent on its velocity at the previous time slot and is restricted by the velocity of the node preceding it on the same lane of the street, as in the Freeway model. Thus, the Manhattan mobility model is also expected to have high spatial dependence and high temporal dependence. However, it provides more freedom than the Freeway model.

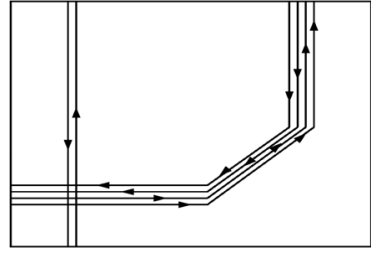

Figure 1. Freeway Model

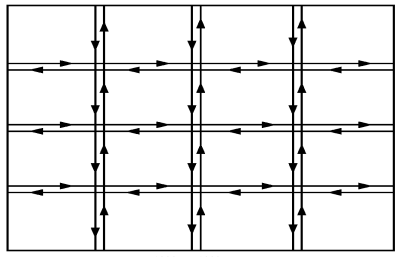

Figure 2. Manhattan Model

\section{Analysis of the effect of node mobility}

To estimate the effect of inaccurate location information caused by node mobility on the geographic routing protocol, we conducted simulations with ns- 2 varying the beacon interval and the maximum speed of mobile nodes for each mobility model. GPSR $[2,11]$ is selected for our simulation because it uses greedy forwarding with face routing, and was shown to perform correctly and efficiently with exact location information. It is a widely accepted protocol for geographic routing in mobile ad hoc and sensor networks. Fifty nodes are placed randomly in a $1500 \mathrm{~m} \times 300 \mathrm{~m}$ field and the combination of beacon intervals of $0.25,0.5,1.0,1.5,3.0,6.0 \mathrm{sec}$ and maximum node speed of $10,20,30,40,50 \mathrm{~m} / \mathrm{sec}$ are simulated. The IMPORTANT mobility tools presented in [9] are used to generate the mobility models. To filter out the noise in simulation results, five different scenarios are generated for each distinct parameter setting and the results represents the average value.

We introduce several metrics to evaluate the performance of the routing protocol in several aspects.

(a) Successful Delivery Rate (SDR): the number of packets successfully delivered to the destination node over the total number of packets transmitted.

(b) Wasted Transmission Rate (WTR): the number of transmission efforts made for dropped packets during the delivery over the total number of packet transmission.

(c) Number of Lost Links (LLNK): the number of lost link problem observed during the packet forwarding.

SDR represents the level of reliability in packet delivery, while WTR represents the level of wasted resources in the network. The latter metric is particularly important when considering energy-constrained wireless networks.

\subsection{Effect of node speed}

The general effect of node speed on the performance of GPSR protocol is similar for all four mobility models. Figure 3 shows the effect of node speed on the performance of GPSR routing protocol. The performance drops as the maximum node speed increases, but the amount of performance drop is different for each mobility model. To see the effect of the node mobility on location-based routing protocol for each mobility model, we calculated the difference between the best value and the worst value of each metric in table 1 . The Manhattan and Freeway models show the biggest performance drop and RWP performs well with increased maximum node speed in the view point of every metric considered. This difference is attributed to the different level of randomness for each mobility model.

If we look at the performance of GPSR itself on various speed levels instead of the amount of performance drop, 
RPGM mobility model consistently outperforms the remaining mobility models in SDR as seen in figure 3.

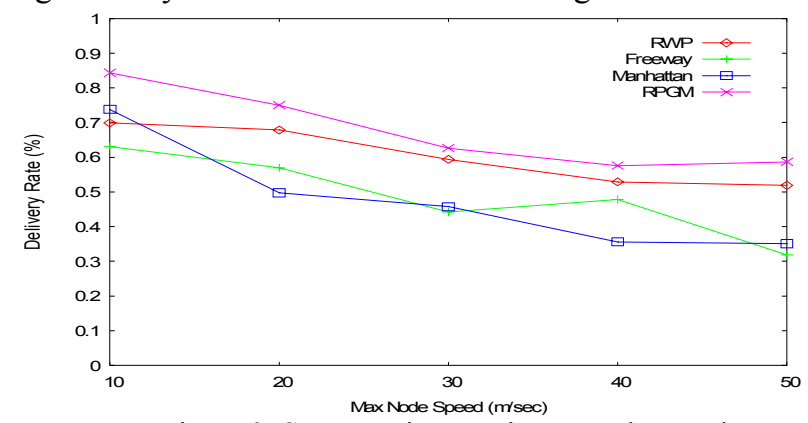

Figure 3. SDR varying maximum node speed

\begin{tabular}{|l|l|l|l|l|}
\hline Difference & RWP & Freeway & Manhattan & RPGM \\
\hline SDR (\%) & 17.9 & 31.2 & 38.7 & 26.7 \\
\hline WTR (\%) & 22.9 & 37.2 & 36.2 & 13.1 \\
\hline LLNK(\#) & 749.6 & 1213.7 & 1258.5 & 475.2 \\
\hline
\end{tabular}

Table 1. The maximum performance difference from varying speed

The average number of LLNKs is consistently lower for RPGM ( $~ 812$ LLNKs) than other mobility models (ranging from 2,366 to 2,586 LLNKs) as we can intuitively expect from the greater correlation between the movements of neighbor nodes, and this explains the better performance of RPGM.

While the faster maximum node movement brings serious performance drop in location-based routing, some interesting results are observed. In figure 4 , we find that the average number of packets delivered via less-than-optimal hops, which is calculated before the actual routing, increased from 5.9 at speed 10 to 19.4 at speed 50, while the ratio of successful packet delivery decreases with high node mobility. This can be considered as a positive effect of node mobility, where the destination node moves towards the source. The result teaches us that the node mobility has both positive and negative influences on geographic routing protocols, and the positive side can be used to improve the performance of packet delivery. This result also supports the necessity of the second part of our suggested mobility prediction scheme, called destination location prediction (DLP).
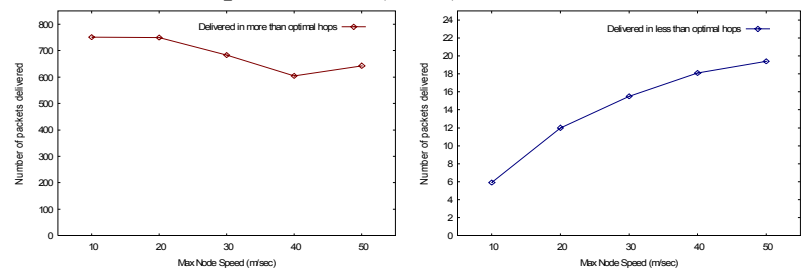

Figure 4. Positive effect of node mobility

\subsection{Effect of beacon interval}

The simulation results on the effect of using different beacon intervals are presented in figure 5, 6. The Performance drop caused by longer beacon interval is smaller $(\sim 12.7$ in SDR) than performance drop by increased mobility ( 28.6).

The simulated geographic routing protocol GPSR performs best when the beacon interval is 0.5 rather than 0.25 , which is the shortest beacon interval we examine. This holds for every metric (SDR, WTR, LLNK) and every mobility model we simulated. When we compare the number of drops for each reason of packet drop between these two beacon intervals, simulations with beacon interval 0.25 shows much more packet drops caused by buffer overflow (indicated by IFQ in the ns-2 [12] trace file).

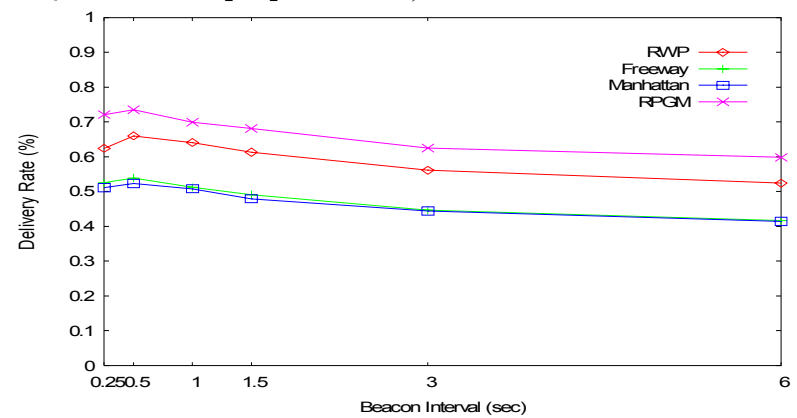

Figure 5. SDR varying beacon interval

\begin{tabular}{|l|l|l|l|l|}
\hline Difference & RWP & Freeway & Manhattan & RPGM \\
\hline SDR (\%) & 13.6 & 12.2 & 10.9 & 13.8 \\
\hline WTR (\%) & 22.6 & 17.5 & 12.2 & 16.7 \\
\hline LLNK(\#) & 722.6 & 837.1 & 636.5 & 235.1 \\
\hline
\end{tabular}

Table 2. Max performance difference by varying beacon interval

Number of drops resulted from other reasons, such as drop by no route (NRTE), by TTL expiration (TTL), by routing loop (LOOP), do not show much difference on the other hand.

This result shows that frequent beacons may cause network congestion and lead to deteriorated performance of geographic routing as well as wastage of network resources.

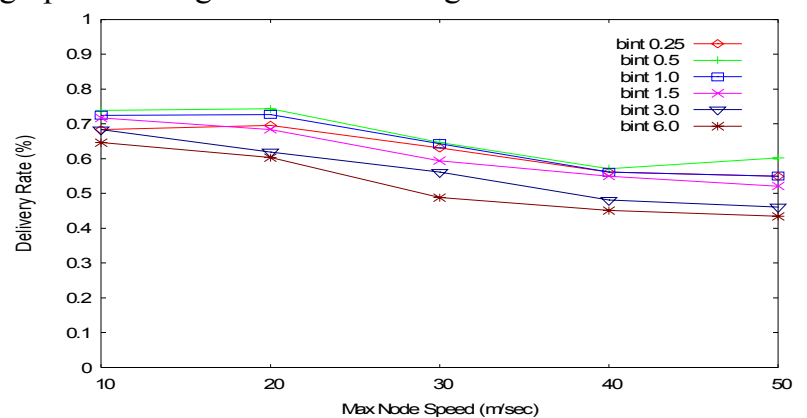

Figure 6. Effect of node mobility, beacon interval (RWP mobility)

\section{Identified problems (caused by mobility)}

Inaccurate location information caused by node mobility produces bad performance of geographic routing protocol as we have shown. Through further analysis we identify two main problems that account for the performance degradation, namely LLNK and LOOP problems, described next.

\subsection{Lost Link (LLNK) problem}

Greedy forwarding mode in GPSR always forwards a packet to the neighbor that is located closest to the destination node. Each node searches its neighbor list to find a node that meets this condition and forwards a packet to this selected next hop neighbor. However, the selected next hop node may not exist within the radio range while it is listed as a neighbor. This situation is defined as lost link (LLNK) problem and can be caused by one of the following two reasons: (1) node mobility: There is a higher probability of packet transmission failure if greedy forwarding is used to forward the packets. With small outward node movement of intended receiver, 
connection between the sender and the receiver can be broken. (2) asymmetry in a communication link: GPSR assumes link symmetry between neighboring nodes. However, this may not be true in many real wireless network environments. Asymmetric communication links exist when there are nodes with different radio ranges, due to environmental effects or node mobility. These problems are illustrated in figure 7.

\subsection{LOOP problem}

With GPSR, a packet is forwarded towards the coordinate of the destination stored in the packet header, and identification of a node is meaningless until the packet reaches the destination node in greedy forwarding. Consider the case when a destination node moves away from its original location and another becomes a node located closest to the original coordinate of the destination. This situation is misunderstood as local maximum by GPSR protocol, and the perimeter mode forwarding is used to resolve this problem. However, packets normally get dropped unless the destination node doesn't come back to near the original location and becomes the closest node to the destination location of the packet. Perimeter forwarding generates wasteful loops in this situation, and we label these situations as LOOP problems, shown in figure 8.

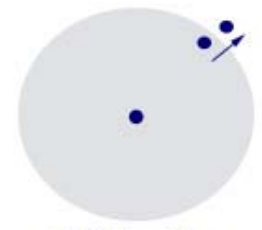

(1) Node mobility

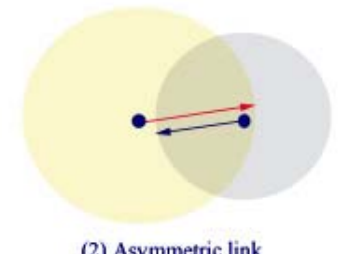

(2) Asymmetric link
Figure 7. Two reasons of LLNK problem

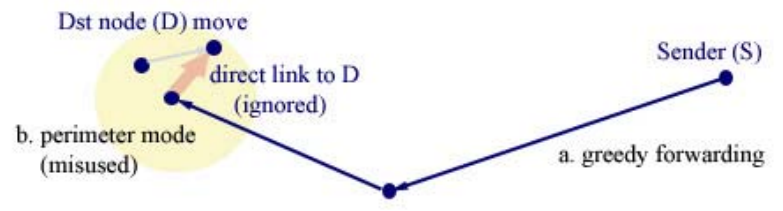

Figure 8. An example case of LOOP problem

\section{MP: Improvement on geographic routing}

We introduce a mobility prediction (MP) scheme for geographic routing that does not require any additional communication or serious calculation. There have been some prior research efforts for mobility prediction. In [13], a mobility prediction scheme in wireless networks and its application to several unicast [14],[15] and multicast [16] routing protocols are introduced. The suggested mobility scheme is employed to calculate the duration of a link connection time. Route expiration time (RET) before the predefined route becomes unavailable can be attained based on the valid link duration, better packet delivery and reduced overhead are achieved.

The mobility prediction scheme in [13] assumes clock synchronization in the network and constant node speed and movement direction. The suggested scheme is effective when nodes exhibit a non-random traveling pattern. Similarly, [17] suggests a mobility prediction scheme that proactively constructs a route for robust and efficient packet delivery. Virtual grid space, where every node stays inside, is introduced and a unique grid-id is given for each grid. The movement pattern of a node is identified based on the previous node movement represented by a sequence of grid-ids stored in the node movement cache. Recent node movement is compared with identified movement pattern via pattern matching to predict the next node movement. Probability of next node movement is calculated and used to cope with node mobility beforehand. Assumptions on virtual grid space and the non-negligible amount of required storage, computation, and communication limit the applicability the proposed scheme.

Our mobility prediction scheme is composed of two prescriptions to identified problems listed in section 4. Suggested schemes are neighbor location prediction (NLP) and destination location prediction (DLP).

\subsection{Neighbor Location Prediction (NLP)}

A neighbor location prediction scheme is introduced as a solution to the $L L N K$ problem. To avoid the bad next-hop node selection, which may result in $L L N K$ problems, the current locations of neighbor nodes are estimated at the moment of packet routing decision with $N L P$. Estimations are based on the recent beacon information received from neighbor nodes. The neighbor list includes the following additional fields for neighbor location estimation: last beacon time ( $L B T)$, node speed in the direction of $\mathrm{x}$-axis $(S x)$ and $y$-axis $(S y)$. When a node receives a new beacon from a neighbor, the current time is stored in $L B T$ together with the location of the neighbor. The beacon receiver searches its neighbor list for previous beacon information from the same neighbor. If previous beacon information from the same neighbor is found in the neighbor list, current node speed of the neighbor, which consists of $S x$ and $S y$, is calculated when it receives a new beacon packet from the same neighbor as follows.

The previous location and beacon time of a neighbor stored in the neighbor list is denoted by $(x 1, y 1, P B T)$ and the same information found in the last beacon packet for the same neighbor is denoted by $(x 2, y 2, L B T)$. The current node speed $S x$ and $S y$ of the neighbor is calculated as follows:

$$
S_{x}=\left(x_{2}-x_{1}\right) /(L B T-P B T) \text { and } S_{y}=\left(y_{2}-y_{1}\right) /(L B T-P B T) \text {. }
$$

The current location of a given neighbor node $\left(X_{\text {est }}, Y_{\text {est }}\right)$ is estimated whenever a node looks up a neighbor list for routing decision based on the calculated node speed and the amount of time passed since LBT:

$$
\begin{gathered}
X_{e s t}=x_{2}+S_{x} *(\text { Current Time }- \text { LBT }) \\
Y_{\text {est }}=y_{2}+S_{y} *(\text { Current Time }- \text { LBT })
\end{gathered}
$$

Our linear location prediction scheme is simple, but very reasonably when the beacon interval and the time since $L B T$ are reasonably small.

Transmission range information of each node is also incorporated in our NLP scheme to avoid the problem caused by asymmetric link resulted from inherent difference in transmission power among deployed nodes. We assume each node knows (or estimates) its approximate radio range and does not forward a packet to a neighbor node that is currently located outside of its range based on the estimated position to avoid $L L N K$. With $N L P$, a packet is forwarded to a neighbor node that meets the following two conditions: 
- A neighbor node that has a closest distance to a destination node from the estimated location of a neighbor node, and

- Distance to a neighbor node is less than the transmission range of a forwarding node.

By incorporating the transmission range information and using the estimated neighbor location information attained from this simple calculation, the $L L N K$ problem identified from previous simulation is greatly reduced for all mobility model in our simulation. The percentages drop in the number of LLNKs is $17.5 \%$ for RWP, $15.2 \%$ for FWY, $14.3 \%$ for $\mathrm{MH}$, and $6 \%$ for RPGM mobility models.

\subsection{Destination Location Prediction (DLP)}

The second part of our mobility prediction scheme is a solution to the LOOP problem, which turns out to be the most serious problem for greedy forwarding. A great number of packets get dropped even when those are delivered to a neighbor node of the destination node. Packet drop after forwarding it to a neighbor of a destination node is the most undesirable thing to do with packet routing because it means more wastage of energy and bandwidth in the network.

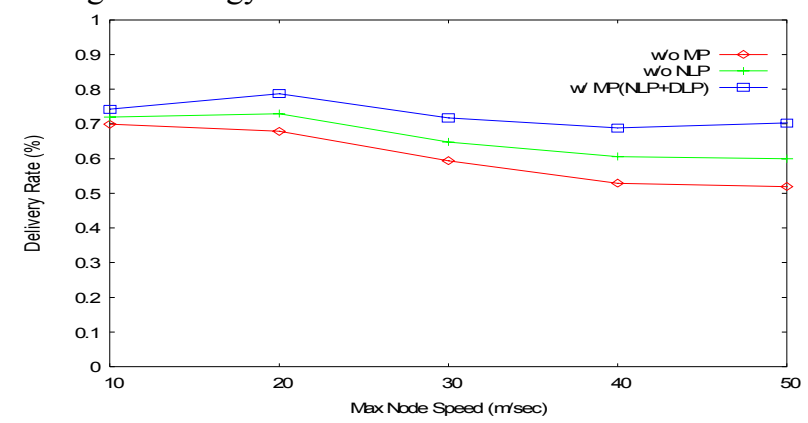

Figure 9. Successful Delivery Rate with various prediction schemes

To avoid this kind of problem and to increase the chance of packet delivery for the case when the destination node is moved out of its original location, a destination location prediction (DLP) scheme is proposed as a second part of MP. With DLP, each node searches its neighbor list for the destination node before it makes a packet forwarding decision based on the location information of the destination. If the destination node exists in the neighbor list and located within the transmission range of the packet holder, the packet is forwarded directly to the destination node without further calculation for finding a closest neighbor to the destination. LOOP problems can be overcome by utilizing the identification information of nodes as well as location information. Figure 9 shows the performance improvement achieved with both NLP and DLP.

Significant amount of lost packets and wasted network resources can be saved by avoiding misjudgement on the local maximum situation. Geographic routing can also utilize the node mobility in a positive way while reducing the problem of it using DLP.

\section{Simulation Results with MP}

With MP (NLP plus DLP), the amount of wasted transmission (WTR) is reduced $18.6 \%$ for RWP, 37.2\% for FWY, $15.2 \%$ for MH, $16.5 \%$ for RPGM, and the SDR levels up even with higher mobility and longer beacon interval. Figure 10 clearly shows the effect of MP on the performance of geographic routing protocol. The impact of faster node movement and infrequent beacon interval has greatly reduced after applying MP to GPSR. SDR is improved $12.3 \%$ for RWP, $26.9 \%$ for FWY, $14.7 \%$ for MH, and $19.8 \%$ for RPGM.

To identify the actual effect of each component in MP, the causes of packet drops in our simulations are analyzed. As discussed earlier, NLP is a scheme to reduce the number of LLNK caused by inaccurate neighbor location information. Broken link connection delays the packet forwarding process in the queue. Packet drops caused by the delay in ARP process (indicated by ARP in the ns- 2 trace file) are closely related to LLNK problem.

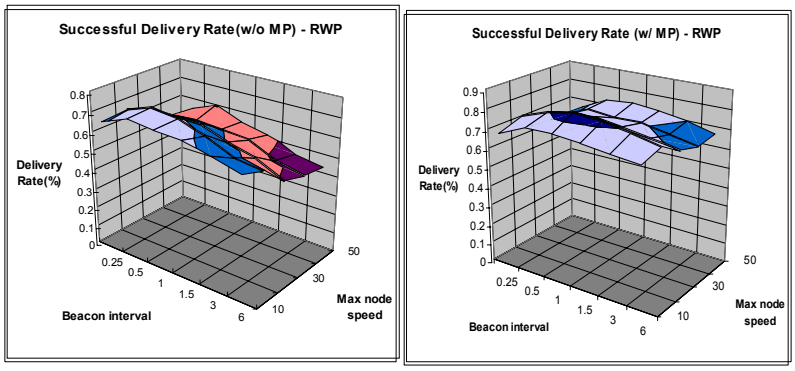

Figure 10. GPSR without MP and with MP

With NLP, the number of packet drop caused by ARP is greatly reduced for every mobility model and for both increased maximum node speed and longer beacon interval cases. We observe noticeable decrease in number of drops by ARP for GPSR with NLP. Examples are shown in figure 11.

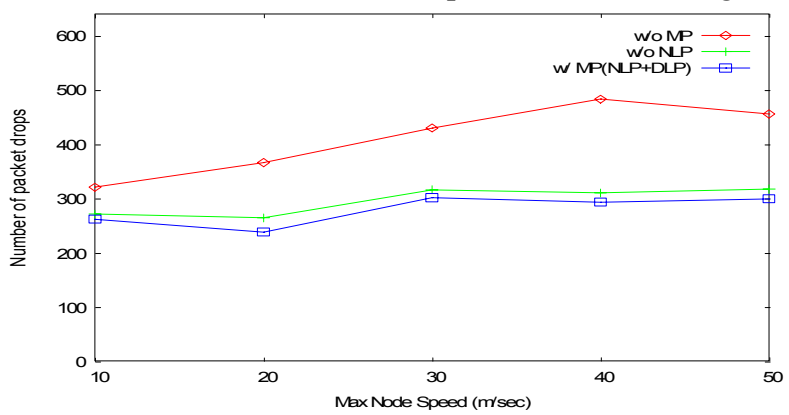

(a) Drop by ARP under RWP mobility

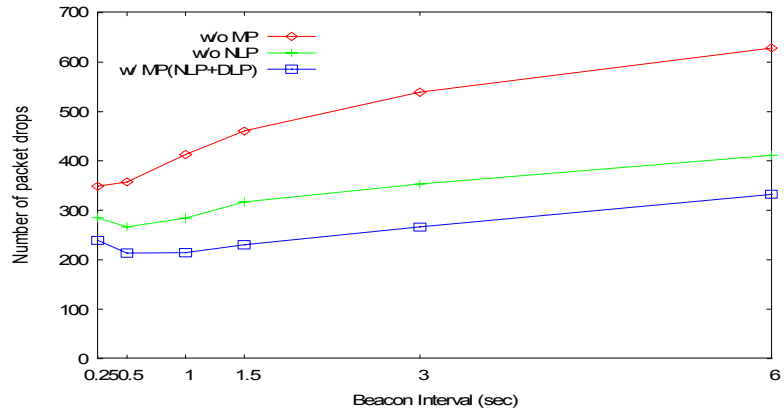

(b) Drop by ARP under Freeway mobility

Figure 11. Number of drops caused by ARP

DLP is a scheme introduced to fix LOOP problem identified in section 4.2. Figure 12 shows example improvements achieved with DLP in the number of packet drops caused by no route (NRTE) and TTL expiration (TTL). Packet drops caused by routing loop (LOOP) and MAC layer callback timer 
(CBK) are also closely related to LOOP problem and exhibit similar improvement in our simulations with DLP.

\section{Conclusion and Future Work}

In this paper, we have presented the effect of inaccurate location information caused by node mobility in geographic routing protocols, and identified two major problems caused by node mobility: LLNK and LOOP problems. We also propose a mobility prediction scheme to address these two revealed problems. For our simulation we chose three main factors, (1) maximum node speed (2) beacon interval (3) mobility pattern that affect the performance of geographic routing to clarify the effect of these factors on the performance of location based routing protocols. The general effects from varying maximum node speeds and beacon intervals are similar for all the studied mobility models. However, the levels of effect are somewhat different. Increased node mobility causes more effect on FWY and MH mobility models. Longer beacon interval deteriorates the performance of RWP and RPGM slightly more. These differences are attributed to the differences between the mobility models.

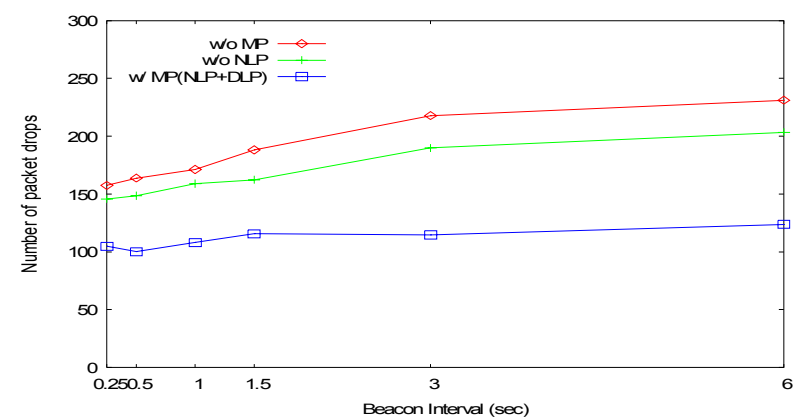

(a) Drop by NRTR under Manhattan mobility

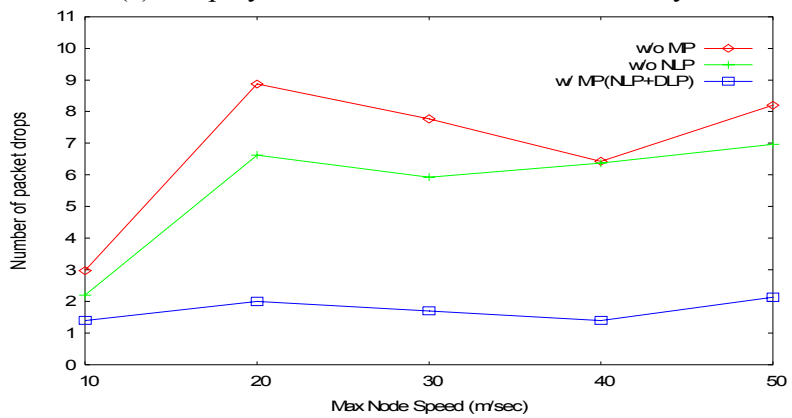

(b) Drop by TTL under RPGM mobility

Figure 12. Number of drops by NRTE and TTL

Both negative side and positive side of node mobility could be found in our simulation results. Identification of two major problems caused by mobility-induced location error and the discovery of the positive effect of node mobility are some of the main contributions of our study. LLNK problem is caused by the movement of neighbor nodes and asymmetry in communication link. LOOP problem is caused by the movement of a destination node. Positive effect of node mobility is utilized by DLP. Our proposed mobility prediction scheme is comprised of neighbor location prediction (NLP) and destination location prediction (DLP) schemes. Each component is introduced to settle down LLNK and LOOP problem. With NLP, the number of lost link problems can be significantly decreased by estimating the actual location of neighbor nodes based on latest movement and by excluding nodes located outside of a sender's radio transmission range. With DLP, unnecessary packet drops near the destination can be avoided, and the positive side of node mobility is exploited as well as negative effect is mitigated.

With the combination of these two schemes in GPSR, the performance in both SDR and WTR is significantly improved. For FWY model we got the best improvement of $27 \%$ more packets are delivered to the destination, and $37 \%$ of wasted transmission effort is reduced with suggested mobility prediction scheme in our simulations.

Other than the saved network resources with MP, we could pursue further saving. As seen in figure 4, the negative effect of increased beacon time is alleviated even with high mobility. Efficient beacon exchange can be achieved with MP when the loss in reliability is less significant than the level of wastage in network resource (e.g., sensor networks).

In our future work, we aim to collect supplementary information from previous node movements to build a more sophisticated mobility prediction schemes. Location estimation scheme will be combined with stability factor for each link to help the sender make better routing decisions and applied for location services [18] as well as other geographic routing protocols.

\section{References}

[1] E. Kaplan. Understanding GPS. Artech House, 1996.

[2] B. Karp and H. T. Kung, "Greedy Perimeter Stateless Routing for Wireless Networks," Mobicom, 2000, pp.243-254.

[3] S. Basagni, I. Chlamtac, V.R. Syrotiuk, and B.A. Woodward, "A distance routing effect algorithm for mobility (DREAM)," Mobicom98.

[4] Y. Ko and N.H. Vaidya, "Location-aided routing (LAR) in mobile an hoc networks," Mobicom, 1998, pp.66-75.

[5] L. Blazevic et al., "Self-Organization in Mobile Ad Hoc Networks: The Approach of Terminodes," IEEE Commun. Mag.,2001.

[6] D. Johnson and D. Maltz, "Dynamic source routing in ad hoc wireless networks," Mobile Computing, pp. 153-181. Kluwer Publishers, 1996.

[7] T. Camp et al., "Performance Comparison of Two Location Based Routing Protocols for Ad Hoc Networks," IEEE INFOCOM, 2002.

[8] V. Park and M. Corson. "A highly adaptive distributed routing algorithm for mobile wireless networks," In Proceedings of INFOCOM, 1997

[9] C. Perkins and E. Royer. "Ad-hoc on-demand distance vector routing," 2nd IEEE Wkshp on Mobile Computing Systems and Apps, p 90-100, Feb99

[10] F. Bai, N. Sadagopan, A. Helmy, "IMPORTANT: A framework to systematically analyze the Impact of Mobility on Performance of RouTing protocols for Adhoc NeTworks," IEEE INFOCOM, pp.825-835, April 2003.

[11] B. Karp, "Geographic Routing for Wireless Networks," PhD Dissertation, Harvard University, October 2000.

[12] L. Breslau et al. "Advances in Network Simulation," IEEE Computer, V.33(N. 5), pp.59-67, May 2000.

[13] W. Su, S.-J, Lee and M. Gerla, "Mobility prediction and Routing in Ad Hoc Wireless Networks," Int'l Jrnl of Network Management, (11) 1, Feb 01.

[14] W. Su and M. Gerla, "IPv6 Flow Handoff in Ad-Hoc Wireless Networks Using Mobility Prediction," IEEE GLOBECOM'99, pp. 271-275.

[15] W. Su, "Motion Prediction in Mobilie/Wireless Networks," PhD Dissertation, UCLA Computer Science Department, Dec. 1999.

[16] S.-J, Lee, W. Su, and M. Gerla, "Ad hoc Wireless Multicast with Mobility Prediction,” IEEE ICCCN'99, Boston, MA, Oct. 1999, pp. 4-9.

[17] Naresh Verma, "Mobility Prediction in Ad Hoc Networks and its application to Routing," Research Workshop, Virginia Tech, May 2002.

[18] S. Giordano and M. Hamdi. "Mobility management: The virtual home region," Technical report, October $1999^{1}$

\footnotetext{
${ }^{1}$ A. Helmy was supported by NSF CAREER, Intel and Pratt\&Whitney grants.
} 